\title{
Analysis on the Optical Characteristics of ZnO Thin Film Prepared by Magnetron Sputtering
}

\author{
Jing-hua Xu, \\ Department of Physics, Tonghua Normal University, Tonghua, Jilin 134002, China; \\ 47837964@qq.com
}

Keywords: magnetron sputtering, ZnO thin films, optical property, morphology

\begin{abstract}
This paper used magnetron sputtering to grow $\mathrm{ZnO}$ film on $\mathrm{Al} 2 \mathrm{O} 3$ substrate. All the samples were characterized by X-ray diffraction (XRD), atomic force microscope (AFM), ultra-violet spectrometer (UVS) and photoluminescence (PL). Results showed that: the doped ZnO thin films were still had the hexagonal wurtzite structure; the magnetron sputtering coated samples' transmission edge would have blue shift and then red shift along with the increase of the annealing temperature; all samples had decent luminescent property; $0^{\circ} \mathrm{C}$ sputtering coated samples had decent surface flatness and even distribution of particles.
\end{abstract}

\section{Introduction}

$\mathrm{ZnO}$ is a wide band-gap semiconductor material with hexagonal wurtzite structure. its band-gap width is approximately $3.37 \mathrm{eV}$, while the exciton binding energy would amount up to $60 \mathrm{meV}$ at room temperature. The big binding energy means that the excitons could give highly effective laser reflection easier, making $\mathrm{ZnO}$ a short wavelength luminescent material used under room temperature or higher temperatures ${ }^{[1,2,3]}$. Magnetron sputtering is a high-speed sputtering technology developed since 1970s. Originally it was used to deposit metal and optical films. With constant improvement of modern technologies, magnetron sputtering has gradually been used to produce semiconductor films $[4,5,6]$. This paper managed to use magnetron sputtering to grow $\mathrm{ZnO}$ thin film on $\mathrm{Al}_{2} \mathrm{O}_{3}$ substrate and then applied annealing treatment at different temperatures, studying the influence of annealing temperature film's structure, ultraviolet transmission, room temperature photoluminescence and surface morphology, etc.

\section{Experiment process:}

\subsection{Substrate cleaning:}

Clean the sapphire substrate for 30 minutes with methylbenzene, deionized water, acetone, deionized water and methylbenzene ultrasonic cleaning in turn. At last, use massive flowing deionized water to rinse the substrate sheet surface and place the substrate sheets in the baking oven where they would be dried and prepared for furtehr usage.

\subsection{Sputtering coating}

The experiment used radio frequency reactive sputtering method to prepare the film. The equipment for film growth used in the experiment was a vacuum multi-target magnetron sputtering coating device manufactured by Beijing Technlo Technology Co., Ltd. The advantages of this device include common sputtering with multiple targets and that the substrate could rotate at an even speed in the film's growing process, helpful to increase the uniformity of the film samples.

The sputtering process's set-up of parameters were: coating time: 2 hours; Ar pressure in the film's growing process: $0.55 \mathrm{~Pa}$; Ar's flow rate: $10 \mathrm{SCCM}$; source power: $80 \mathrm{~W}$; rotating speed of substrate: $10 \mathrm{r} / \mathrm{min}$.

\subsection{Annealing treatment}

Thermal annealing is a commonly used technology in preparing film materials. Film samples obtained in magnetron sputtering would leave internal stress inside the film which would lead to some defects such as coarse grains, banded structure and segregation, etc. To improve the film quality 
and remove the defects, annealing treatment is usually required to obtain the most outstanding film properties.In this experiment, the samples were placed in a muffle furnace for annealing. All samples were annealled at $800^{\circ} \mathrm{C}, 900^{\circ} \mathrm{C}, 1000^{\circ} \mathrm{C}$ and $1100^{\circ} \mathrm{C}$ for $1 \mathrm{~h}$. then samples would cool down with the furnace until reaching the room temperature, so a series of $\mathrm{ZnO}$ thin films were produced.

\section{Results and Discussion}

\subsection{The crystal structure of La -doped $\mathrm{ZnO}$ thin films}

The film's structure and growing quality studied by X-ray diffractometer. Fig. 1 is the XRD diffraction spectrums under different annealing temperatures showing that the ZnO film samples were of hexagonal wurtzite structure. All samples's XRD spectral lines had six diffraction peaks, respectively corresponding to ZnO's diffraction peaks of (100), (002), (101), (102), (110) and (103). All of the diffraction peaks had no obvious preferred orientation. The diffraction peaks were all very wide, a possible result of wide range of particle size distribution.It can be seen that all $\mathrm{ZnO}$ film samples showed diffraction peaks (100), (002) and (101) with basically matching intensity of peaks. As the annealing temperature rose, the intensity of the three peaks would increase first and then decrease later by a small margin. We can been seen that samples subject to annealing treatment under $1000^{\circ} \mathrm{C}$ had the best crystal structure.

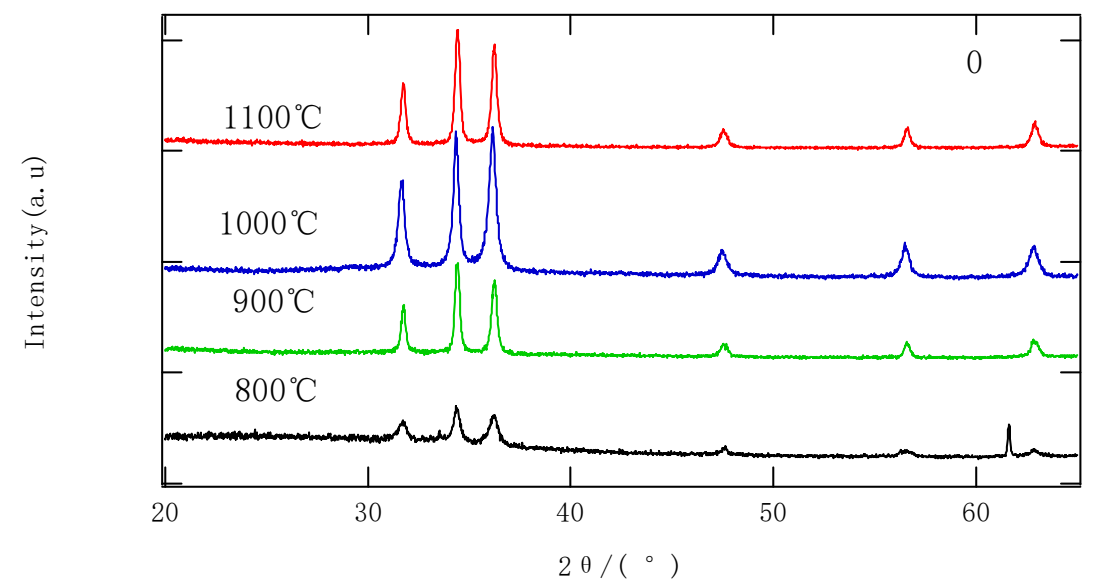

Fig. 1 XRD spectrum of samples

\subsection{Analysis on ultraviolet transmittance spectrum (UVS)}

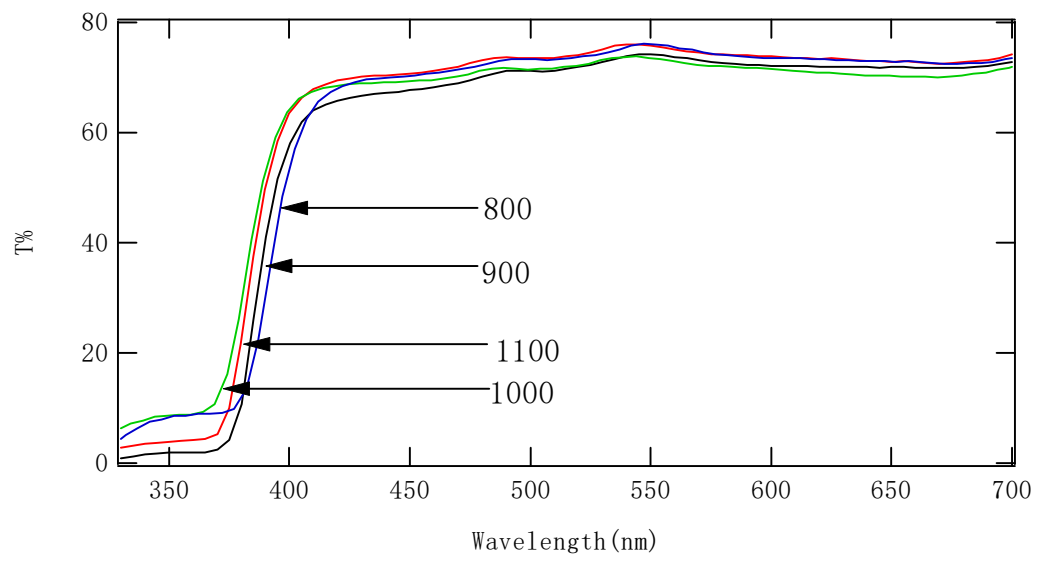

Fig. 2 Samples' transmittance spectrum under room temperature

From Fig. 2 we can see that all samples have a transmissivity decline sharply around 380nm, corresponding to the intrinsic lighting region of $\mathrm{ZnO}$ film samples. It can be seen that with rising annealing temperature, the samples' transmittance edges would show blue shift first and red shift later, indicating that along with the rise of the annealing temperature more defects would be filled, leading to an improved crystalline property of $\mathrm{ZnO}$. Therefore, the optical gap would be narrowed, which is shown in the transmittance rate spectrum as the transmittance edges shifting toward the short wavelength direction, an occurrence consistent with the description of relevant literature[7].With 
continuous rise of the annealing temperature, $\mathrm{ZnO}$ would break down and form defects which would promote the red shift of the absorbing edges.

\subsection{Photoluminescence spectrum (PL) analysis}
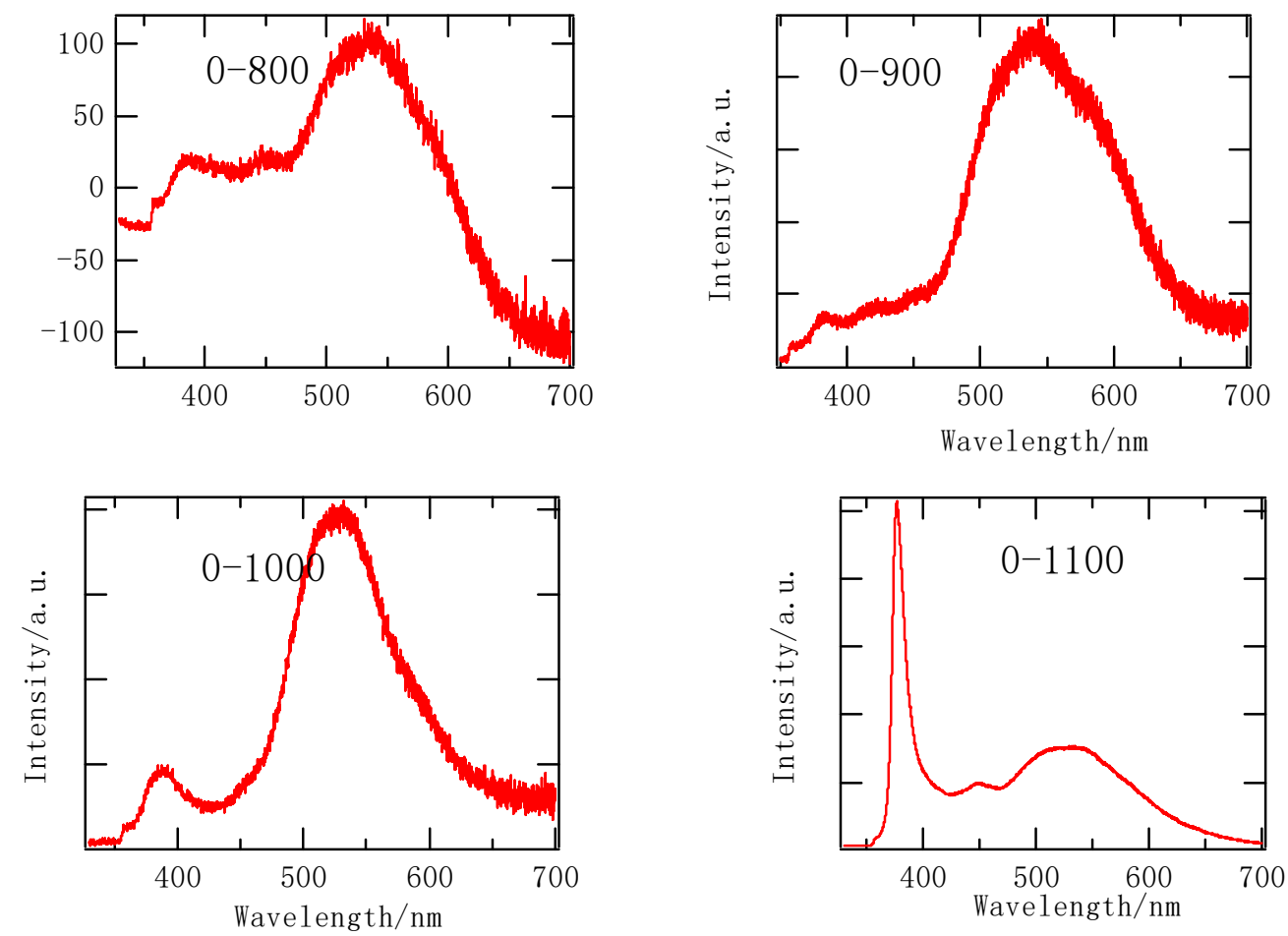

Fig. 3 PL spectrum of the samples

Fig. 3 is the low-temperature photoluminescence spectrum of the film samples at the annealed temperature from $800^{\circ} \mathrm{C}$ to $1100^{\circ} \mathrm{C}$, respectively. From the spectrum we can see that the samples had decent luminscent property. Under different annealing temperatures, samples had ultraviolet intrinsic luminscent peak around 380 and defective luminescence of yellow and green light. Under the annealing temperature of $1100^{\circ} \mathrm{C}$, samples had the strongest intrinsic blue-purple luminescence. This luminescence was generally considered to have been caused by the emission of near-band edges (NBE). The yellow-green defective luminscence of the samples were strong under other temperatures, a result generally considered to be related to the composite of free excitons[8]. This luminscent peak was mainly caused by the defect ofdeep energy levels, as the massivedefects of $\mathrm{ZnO}$ after annealing treatment would lead to highly intensive luminescent peaks, thus reducing the $\mathrm{ZnO}$ 's characteristic peak at $3.37 \mathrm{eV}$. This indicates that it is not that higher annealing temperature would lead to better optical performance of the film samples.

\subsection{Analysis on surface morphology (AFM)}

The film's surface morphology not only influences the sample's physical and chemical property but also has internal connections with the film's preparing conditions and crystal structure, etc. Surface morphology has important influence on transparent conductive film's optical properties and electric properties. This paper used the SPII-3800 model of atomic force microscope as manufactured by Seiko to test the surface morphology of the samples. The test results were as follow: the sputtering samples at four different temperatures all assumed particle-like morphology with quite even distribution of surface particles. However, different annealing temperatures cause different sizes of particles, with particle size under the annealing temperature of $900^{\circ} \mathrm{C}$ showing the maximum particle size. 

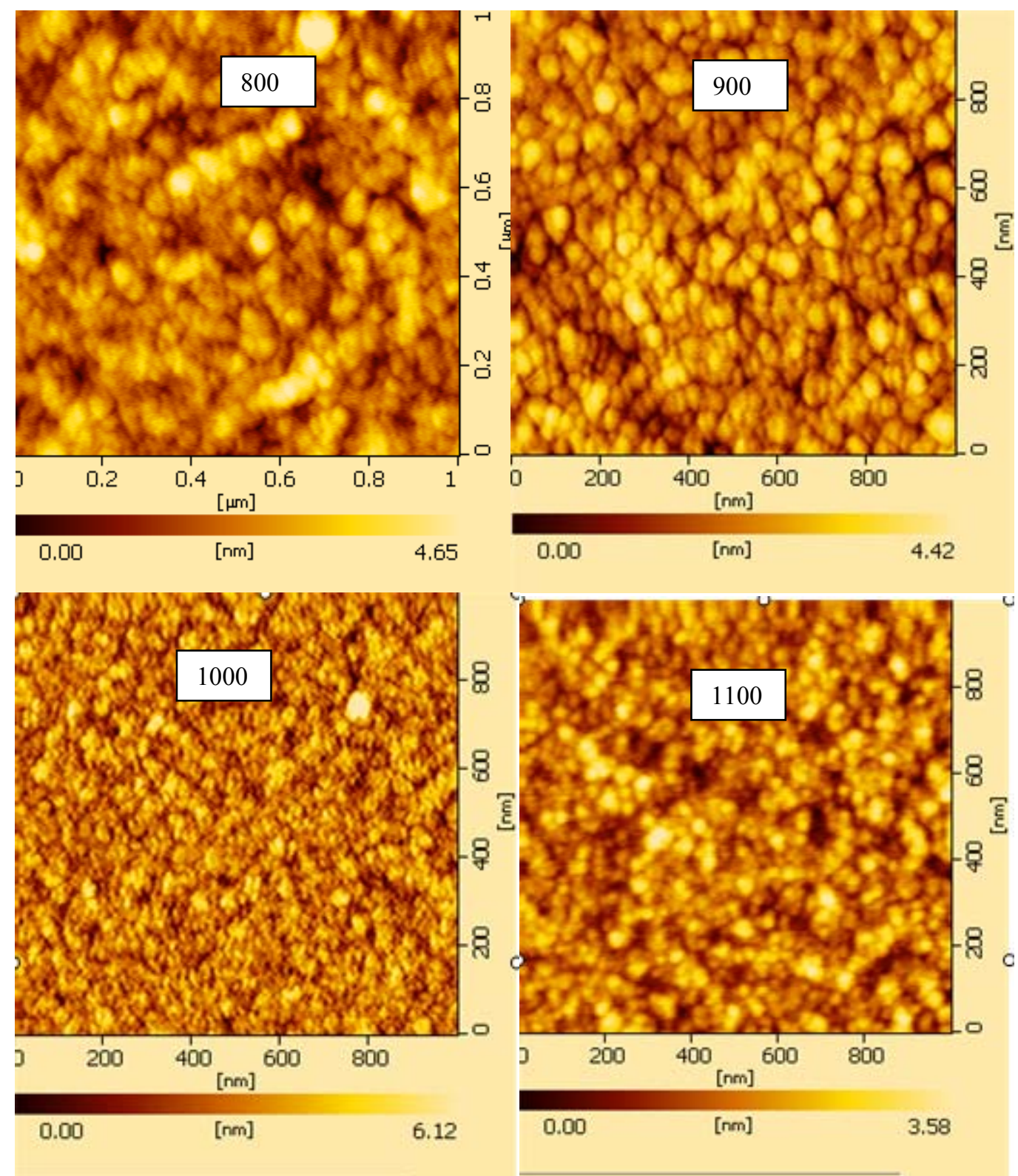

Fig. 4 AFM morphology of samples

\section{Conclusions}

The magnetron sputtering coating samples' transmittance edges would show blue shift and then red shift with increased annealing temperatures. All samples had decent luminescent property. Under the annealing temperature of $1100^{\circ} \mathrm{C}$, samples had good ultraviolet luminescent property; under the annealing temperatures of $800^{\circ} \mathrm{C}, 900^{\circ} \mathrm{C}$ and $1000^{\circ} \mathrm{C}$, the samples had very good defective luminescent property. Under the annealing temperature of $900^{\circ} \mathrm{C}$, the sample surface had the best flatness of surface morphology and even distribution of particles.

\section{References}

[1]. Fei Gao,Xiao Yan Liu: Microstrcture and opticl properties of Fe-doped ZnO thin films prepared by DC magnetron sputtering [J] .Journal of Crystal Growth. 2013， 371 126-129

[2]. V Srikant. D.R. Clarke. On the optical band gap of zinc oxide [J] . Appl. Pbys. 1998, 83, 1447-1451. 
[3]. Lv Jianguo, Ye Zhizhen, Chen Hanhong, et al. DC Response Magnetron Sputtering Growth P-type ZnO Films and Study on Their Properties [J]. Vacuum Science and Technology, 2003, 23(1): 5-8.

[4]. Wen Jun, Chen Changle. RF Sputtering Neodymium-mixed ZnO Film's Structure and Luminescent Property [J]. Chinese Journal of Luminescence 2008, 29 (5) 856-861.

[5]. T. M. Williams, D. Hunter, and A. K. Pradhan, Photoinduced piezo-optical effect in Er doped ZnO films [J]. Applied Physics Letters 2006, 89, 043116.

[6]. Soumahoro, Colis,Schmerberm.Structural,otical,spectroscopic and electrical properties of Mo-doped $\mathrm{ZnO}$ thin films grown by radio freuency magnetron sputtering. [J] Thin Solid Films.(2014, 566, 61-69

[7]. Zhang Ruifang, Theoretical and Experimental Study on Adjustment and Control of Rare Earth on ZnO's Energy Gap [D]. Inner Mongolia: Chemistry and Chemical Engineering School of Inner Mongolia University, 2007.

[8]. Du Jilong, Preparation of and Study on P-type ZnO. [D] Suzhou: Suzhou University. 2010. 